BMJ

Open

Gastroenterology

\section{Systemic sclerosis is associated with specific alterations in gastrointestinal microbiota in two independent cohorts}

To cite: Volkmann ER, Hoffmann-Vold A-M, Chang Y-L, et al. Systemic sclerosis is associated with specific alterations in gastrointestinal microbiota in two independent cohorts. BMJ Open Gastro 2017;3: e000134. doi:10.1136/ bmjgast-2017-000134

- Additional material is available. To view please visit the journal (http://dx.doi.org/ 10.1136/bmjgast-2017000134)

ERV and A-MH-V shared first authorship.

Received 20 January 2017 Revised 16 March 2017 Accepted 20 March 2017
For numbered affiliations see end of article.

Correspondence to Dr Elizabeth R Volkmann; evolkmann@mednet.ucla.edu

\section{ABSTRACT}

Objective: To compare faecal microbial composition in patients with systemic sclerosis (SSc) from 2 independent cohorts with controls and to determine whether certain genera are associated with SScgastrointestinal tract (GIT) symptoms.

Design: Adult patients with SSc from the University of California, Los Angeles (UCLA) and Oslo University Hospital (OUH) and healthy controls participated in this study (1:1:1). All participants provided stool specimens for $16 \mathrm{~S}$ rRNA sequencing. Linear discriminant analysis effect size demonstrated genera with differential expression in SSc. Differential expression analysis for sequence count data identified specific genera associated with GIT symptoms as assessed by the GIT 2.0 questionnaire.

Results: The UCLA-SSC and OUH-SSC cohorts were similar in age (52.1 and 60.5 years, respectively), disease duration (median (IQR): 6.6 (2.5-16.4) and 7.0 (1.0-19.2) years, respectively), gender distribution ( $88 \%$ and $71 \%$, respectively), and GIT symptoms (mean (SD) total GIT 2.0 scores of $0.7(0.6)$ and 0.6 (0.5), respectively). Principal coordinate analysis illustrated significant microbial community differences between SSc and controls (UCLA: $p=0.001$; OUH: $\mathrm{p}=0.002$ ). Patients with SSc had significantly lower levels of commensal genera deemed to protect against inflammation, such as Bacteroides (UCLA and OUH), Faecalibacterium (UCLA), Clostridium (OUH); and significantly higher levels of pathobiont genera, such as Fusobacterium (UCLA), compared with controls. Increased abundance of Clostridium was associated with less severe GIT symptoms in both cohorts.

Conclusions: The present analysis detected specific aberrations in the lower GIT microbiota of patients with SSc from 2 geographically and ethnically distinct cohorts. These findings suggest that GIT dysbiosis may be a pathological feature of the SSc disease state.

\section{INTRODUCTION}

The majority of patients with systemic sclerosis (SSc) experience gastrointestinal tract (GIT) dysfunction. ${ }^{1} 2$ Symptoms of lower

\section{Summary box}

What is already known about this subject?

- Gastrointestinal tract dysfunction affects over $90 \%$ of patients with systemic sclerosis.

- The pathogenesis of lower gastrointestinal tract dysfunction in systemic sclerosis is largely unknown.

- Emerging evidence suggest that gastrointestinal tract dysbiosis may be a feature of the systemic sclerosis disease state.

What are the new findings?

- The study found specific alterations in the gastrointestinal microbiota in two independent systemic sclerosis cohorts.

- The extent of dysbiosis (ie, alterations in the intestinal microbiota) appeared greatest in patients from the American systemic sclerosis cohort compared with those from the Norwegian systemic sclerosis cohort.

- Specific genera were associated with severity of gastrointestinal tract symptoms.

How might it impact on clinical practice in the foreseeable future?

- Currently, few effective treatment options exist for managing lower gastrointestinal tract symptoms in patients with systemic sclerosis. If specific genera are found to contribute the gastrointestinal tract phenotype in systemic sclerosis, such genera could provide specific targets for intervention to avert or treat this important clinical dimension of systemic sclerosis.

GIT involvement ${ }^{3}$ adversely affect quality of life and social functioning. ${ }^{45}$ Unfortunately, no effective treatment options exist for eliminating these disruptive symptoms, largely because the pathogenesis of this dimension of SSc is poorly understood.

GIT dysbiosis occurs in a number of chronic inflammatory conditions, including inflammatory bowel disease (IBD) ${ }^{6-9}$ and 
recent studies suggest that alterations in GIT microbiota may be a feature of the SSc disease state. ${ }^{3}{ }^{10}$ Our prior study demonstrated that patients with SSc had decreased abundance of beneficial human commensal genera known to produce key energy metabolites and antiinflammatory molecules for mucosal health (eg, Faecalibacterium; Clostridium) with a concurrent increase in potential pathobiont genera (eg, invasive $\gamma$ Proteobacteria; Fusobacterium), compared with controls. ${ }^{10}$

This study also demonstrated that increased abundance of Clostridium and decreased abundance of Fusobacterium were independently associated with decreased GIT symptoms. ${ }^{10}$ To further explore whether alterations in microbial composition may serve as a pathogenic factor in SSc-GIT dysfunction, the present study examined the lower GIT microbiota from two independent SSc cohorts. Whereas our prior study examined colonic mucosal microbes via colonoscopy, the present study examined stool specimens from the same patients with SSc. ${ }^{10}$ Assessment of stool specimens may reflect other aspects of the gut microbiota (eg, metabolic features) and can be obtained by a less invasive approach.

The objectives of this study, not previously explored in the SSc literature, were to: (1) establish whether analysis of stool specimens can distinguish the GIT microbiota of patients with SSc versus healthy controls; (2) compare the GIT microbiota of patients with SSc from two geographically independent cohorts; and (3) evaluate the hypothesis that specific microbial genera are associated with SSc-GIT symptom severity.

\section{MATERIALS AND METHODS \\ Study participants}

Patient participants were consecutively enrolled from the outpatient rheumatology clinics at University of California, Los Angeles (UCLA) and Oslo University Hospital (OUH). Eligible participants included adult ( $\geq 18$ years) patients with SSc according to the 2013 American College of Rheumatology/European League Against Rheumatism Classification Criteria for SSc. ${ }^{11}$ Exclusion criteria included IBD, inability to withstand from taking an antibiotic and a probiotic for at least 3 weeks prior to the stool collection. Patients were allowed to continue taking a proton pump inhibitor medication because this agent exerts negligible effects on colonic microbiota. ${ }^{12}$

Healthy controls stool specimens were obtained from the UCLA Specialized Center of Research in Neurovisceral Sciences and Women's Health Repository, which consists of stool specimens from healthy adult participants (12 men and 9 women) who do not have GIT symptoms. These control participants were different than the control participants used in our prior study. ${ }^{10}$ We attempted to match 17 of these healthy participants by age and sex with the UCLA-SSc cohort patients (1:1); however, the healthy control cohort had more men than women so gender matching was not always possible. These healthy controls specimen also served as the controls for the OUH-SSc cohort.

The UCLA Institutional Review Board and the Regional Committee of Health and Medical Research Ethics in Norway approved the study protocol and written informed consent was obtained from each participant.

\section{Specimen procurement and processing}

Participants at both sites collected stool specimens using our previously published, standard collection method ${ }^{13}$ and immediately froze the specimen. Frozen specimens were subsequently transferred on ice to the participant's study centre and stored at $-80^{\circ} \mathrm{C}$. OUH specimens were shipped overnight on dry ice to UCLA for further processing. Please see the online supplementary appendix for complete details of specimen procurement and processing.

\section{S rRNA gene sequencing and microbial composition analysis}

The microbiota from the stool specimens were profiled by multiplex sequencing for bacterial rRNA genes using an Illumina HiSeq 2500 (Illumina, San Diego, California, USA) sequence technique. All samples (UCLA-SSc, OUH-SSc, controls) were analysed simultaneously at UCLA to avoid any batch effects. The exact details of this approach have been outlined in our prior publication, ${ }^{9} 10$ and are summarised in the online supplementary appendix.

\section{Faecal calprotectin}

Faecal calprotectin concentrations were measured in duplicate from the stool specimens of the UCLA-SSc and healthy control participants using a commercial kit (KTR-849, Epitope Diagnostics, San Diego, California, USA) according to the manufacturer's instructions.

\section{Assessment of GIT symptoms}

On the day of their stool collection, the UCLA and OUH-SSc participants completed the GIT 2.0, a valid measure of GIT symptom severity in patients with SSc. ${ }^{14}$ The questionnaire consists of seven domains and has been translated and validated in several languages, including Norwegian. scores on the GIT 2.0 can indicate self-rated severity (ie, none/mild vs moderate vs severe/ very severe disease) of GIT involvement based on previously published score thresholds. ${ }^{14}$

\section{Statistical and bioinformatics analyses \\ Statistical approach}

Analyses were performed using R V.3.1.2. Mean and SDs were used to describe continuous parametric data; median and IQRs were used to describe continuous non-parametric data. All tests were two-sided with a 0.05 $\alpha$ level. To compare the microbial communities of SSc versus control samples, $\alpha$ and $\beta$ diversity were analysed. The $\alpha$ diversity represents the complexity of composition within members of a group, and $\beta$ diversity represents 
the between-participant similarity of microbial composition and enables the identification of differences between samples within a group. We also examined taxonomic differences at specific levels (ie, phylum, genus) using linear discriminant analysis effect size (LefSe). We used the false discovery rate (FDR) of Benjamini and Hochberg ${ }^{15}$ and a significant association was defined at the FDR q-value $\leq 0.1$. Please see the online supplementary appendix for further details.

\section{RESULTS}

\section{Participant characteristics}

Seventeen UCLA-SSc cohort patients (88\% women; median age 52.1 years) and 17 OUH-SSc cohort patients
(71\% women; median age 60.5 years) were enrolled (table 1). The healthy control cohort $(\mathrm{N}=17)$ was younger than the SSc cohorts (median age 29.0 years), and had a predominance of women ( $60 \%$ women). The OUH-SSc cohort was mostly Caucasian (94\%); whereas the UCLA-SSc and healthy control cohorts had more Hispanics $(35 \%$ and $24 \%$, respectively, vs $6 \%$ in the OUH-SSc cohort). The mean body mass index (BMI) was similar in the two SSc cohorts $(\sim 24$ for both cohorts) and slightly higher in the controls (28.1), although the difference in BMI between SSc participants and healthy controls was not significant.

UCLA-SSc and OUH-SSc cohort patients had similar SSc disease durations, proportion of patients with diffuse cutaneous sclerosis, and autoantibody profiles (table 1 ).

\section{Table 1 SSc participant characteristics}

\begin{tabular}{|c|c|c|}
\hline & UCLA-SSc participants $(\mathrm{N}=17)$ & OUH-SSc participants $(\mathrm{N}=17)$ \\
\hline Age (years) & Median 52.1 (IQR 46.6-63.0) & 60.5 (IQR 46.0-71.0) \\
\hline Female & $15(88.2 \%)$ & $12(70.6 \%)$ \\
\hline \multicolumn{3}{|l|}{ Race } \\
\hline White & $9(52.9 \%)$ & $16(94.1 \%)$ \\
\hline Asian & $2(11.8 \%)$ & 0 \\
\hline More than one race & $4(23.5 \%)$ & 0 \\
\hline Other & $2(11.8 \%)$ & 0 \\
\hline Hispanic & $6(35.3 \%)$ & $1(5.9 \%)$ \\
\hline BMI & $24.0(4.2)$ & $24.5(3.6)$ \\
\hline Diffuse cutaneous disease & $6(35.3 \%)$ & $7(41.2 \%)$ \\
\hline SSc disease duration (years) & Median 6.6 (IQR 2.5-16.4) & Median 7.0 (IQR 1.0-19.2) \\
\hline Early SSc (<3 years) & $4(23.5 \%)$ & $7(41.2 \%)$ \\
\hline ANA positive & $15 / 16(93.8 \%)$ & $17 / 17(100 \%)$ \\
\hline Scl-70 positive & $3 / 11(27.3 \%)$ & $4 / 17(23.5 \%)$ \\
\hline Anticentromere positive & $5 / 11(45.5 \%)$ & $9(52.9 \%)$ \\
\hline HRCT-defined interstitial lung disease & $12 / 17(70.6 \%)$ & $8 / 17(47.1 \%)$ \\
\hline Current prednisone use* & $3(17.6 \%)$ & $2(11.8 \%)$ \\
\hline Current other immunosuppressant use $†$ & $6(35.3 \%)$ & $2(11.8 \%)$ \\
\hline Current use of probiotic oral supplement & $3(17.6 \%)$ & $3(17.6 \%)$ \\
\hline Current use of proton pump inhibitor & $10(58.8 \%)$ & $5(29.4 \%)$ \\
\hline \multicolumn{3}{|l|}{ Current SSc disease activity } \\
\hline MRSS $>18$ & $3(17.6 \%)$ & $5(29.4 \%)$ \\
\hline Increase in MRSS & $2(11.8 \%)$ & $3(17.6 \%)$ \\
\hline Digital ulcers & $4(23.5 \%)$ & $4(23.5 \%)$ \\
\hline Tendon friction rubs & $2(11.8 \%)$ & $3(17.6 \%)$ \\
\hline DLCO $<70 \%$ predicted & $10(58.8 \%)$ & $8(47.1 \%)$ \\
\hline GIT 2.0 total score & Mean $0.7(0.6) \S$ & Mean $0.6(0.5) \S$ \\
\hline Distension/bloating & Mean $1.5(0.9) \S$ & Mean $1.2(0.8) \S$ \\
\hline Diarrhoea & Mean $0.4(0.6) \rrbracket$ & Mean $0.3(0.3) \emptyset$ \\
\hline Faecal soilage & Mean $0.5(0.9) \rrbracket$ & Mean $0.3(0.6) \rrbracket$ \\
\hline Constipation & Mean $0.7(0.7) \S$ & Mean $0.6(1.1) \S$ \\
\hline Emotional well-being & Mean $0.5(0.7) \S$ & Mean $0.5(0.7) \S$ \\
\hline Social functioning & Mean $0.5(0.5) \S$ & Mean $0.4(0.6) \emptyset$ \\
\hline \multicolumn{3}{|c|}{$\begin{array}{l}\text { Values are } n(\%) \text {, except where otherwise noted. } \\
{ }^{*} \text { Dosages of prednisone were } \leq 10 \text { mg daily. } \\
\text { tImmunosuppressant medications used included mycophenolate (UCLA: } N=1 ; \text { OUH: } N=2) \text { and azathioprine (UCLA: } N=2) \text {. } \\
\text { fProbiotic used in the UCLA-SSc cohort included culturelle }(\mathrm{N}=1) \text {, florify }(\mathrm{N}=1) \text { and align }(\mathrm{N}=1) \text {. For the OUH-SSc cohort, patients consumed } \\
\text { probiotic enriched sour milk products }(\mathrm{N}=3) \text {. Probiotics were not consumed within } 3 \text { weeks of the stool collection. } \\
\text { §Score indicates moderate symptom severity. }{ }^{14} \\
\text { ๆScore indicates mild symptom severity. }{ }^{14} \\
\text { ANA, anti-nuclear antibody; BMI, body mass index; DLCO, diffusing capacity for carbon monoxide; GIT, gastrointestinal tract; HRCT, } \\
\text { high-resolution CT; MRSS, Modified Rodnan Skin Score; OUH, Oslo University Hospital; SSc, systemic sclerosis; UCLA, University of } \\
\text { California, Los Angeles. }\end{array}$} \\
\hline
\end{tabular}


However, more patients in the UCLA-SSc cohort (71\%) had clinically significant interstitial lung disease (ILD) by high-resolution CT, ${ }^{16}$ compared with the OUH-SSc cohort $(47 \%)$.

The UCLA-SSc and OUH-SSc cohorts also had similar GIT symptom profiles (table 1). The mean GIT 2.0 scores indicated moderate symptom severity ${ }^{14}$ for the total score, as well as for the following domains: distension/bloating, emotional well-being and constipation (table 1). The mean GIT 2.0 scores for faecal soilage and diarrhoea indicated mild symptom severity ${ }^{14}$ (table 1). Six UCLA-SSc participants (35\%) had taken antibiotics in the 3 months preceding stool collection; the mean time between cessation of antibiotics and stool collection was 6.5 weeks (range 4-12 weeks). None of the OUH-SSc cohort patients had taken antibiotics 3 months prior to stool sampling.

Only three of the UCLA-SSc cohort patients had elevated levels of faecal calprotectin, which was defined as $>50 \mu \mathrm{g} / \mathrm{g}$. None of the control participants had elevated levels of faecal calprotectin.

\section{Colonic microbial diversity in SSc}

After the operational taxonomic unit (OTU) selection process, a total of 231, 250 and 184 species-level OTUs were generated from the UCLA-SSc, OUH-SSc and healthy control cohorts, respectively. The $\alpha$ diversity (ie, the complexity of microbial composition) was similar between the UCLA-SSc and control participants (see online supplementary figure S1) and between the OUH-SSc and control participants (see online supplementary figure S2). There were also no significant differences in $\alpha$ diversity between the UCLA-SSc and OUH-SSc participants (see online supplementary figure S3).
The $\beta$ diversity was then computed to determine whether SSc and control participants differed in their microbial composition. The principle coordinate analysis visualisation of the weighted Unifrac distances and analysis of variance using distance matrices (Adonis) of this comparison are shown in figure 1. In the UCLA-SSc $\left(\mathrm{R}^{2} \quad 0.355 ; \mathrm{p}=0.001\right)$ and the OUH-SSc $\left(\mathrm{R}^{2} \quad 0.126\right.$; $\mathrm{p}=0.002$ ) cohorts, the microbial composition among patients with SSc was significantly different than healthy controls. The magnitude of this difference appeared greater between the UCLA-SSc cohort and healthy controls than between the OUH-SSc cohort and healthy controls based on the $\mathrm{R}^{2}$ values and as illustrated in figure 1 . The $\beta$ diversity also differed between the UCLA-SSc and OUH-SSc cohort patients $\left(\mathrm{R}^{2} 0.145\right.$; $\mathrm{p}=0.002)$.

\section{Colonic microbial genera are differentially abundant in patients with SSc}

To begin to define the compositional differences between patients with $\mathrm{SSc}$ and healthy controls predicted by the $\beta$ diversity analysis, the relative abundances of microbial composition at different taxonomic levels were computed (figure 2). The two predominant phyla in SSc samples were Bacteroidetes (UCLA: 21.3\%; OUH: 45.0\%; controls: 63.2\%), and Firmicutes (UCLA: 63.5\%; OUH: $42.8 \%$; controls: $33.0 \%$ ). The relative abundance of Bacteroidetes was significantly decreased in the UCLA-SSc cohort compared with the healthy control cohort $(p<0.0001)$ and in the OUH-SSc cohort compared with the healthy control cohort $(\mathrm{p}=0.009)$.

LefSe multivariate analysis demonstrated significant taxonomic differences between SSc and healthy controls at the genus level for the UCLA-SSc (figure 3) and OUH-SSc (figure 4) cohorts. The LefSe analysis comparing the UCLA-SSc and healthy control cohorts yielded
Figure 1 Significant differences in the $\beta$ diversity of the SSc and healthy samples as demonstrated by principal coordinate analysis plots of the weighted UniFrac distance. Each dot represents a sample from a UCLA-SSc cohort patient (open circle) or a healthy control (closed circle). Each star represents a sample from a OUH-SSc cohort patient. The $p$ values provided were calculated by analysis of variance using distance matrices. OUH, Oslo University Hospital; PCoA, principle coordinate analysis; SSc, systemic sclerosis; UCLA, University of California, Los Angeles.

\section{Weighted PCoA Plot}

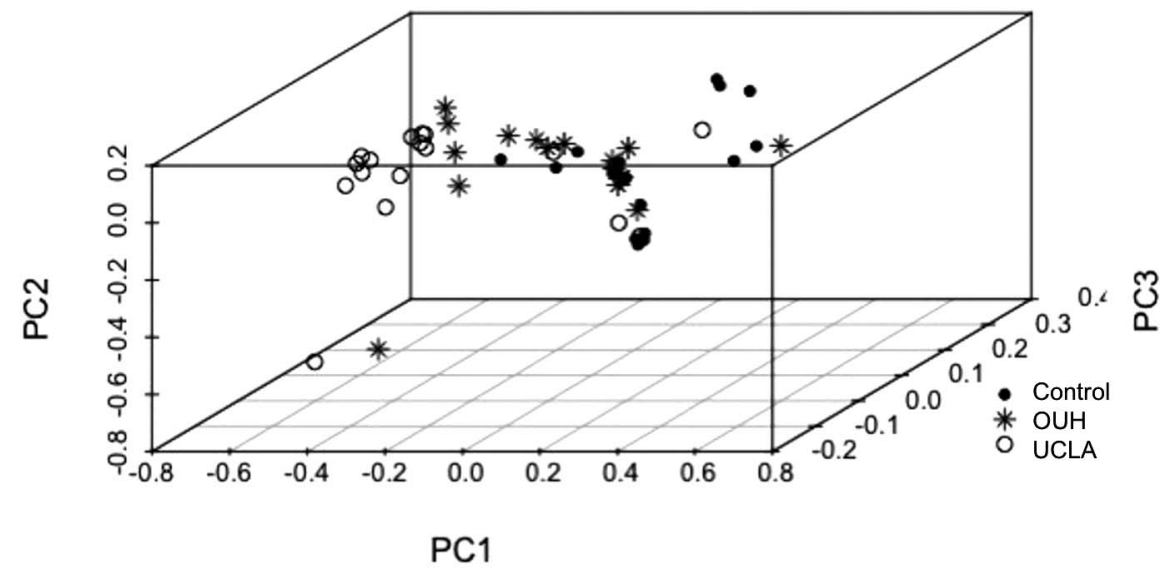

Control vs OUH:

Control vs UCLA: 

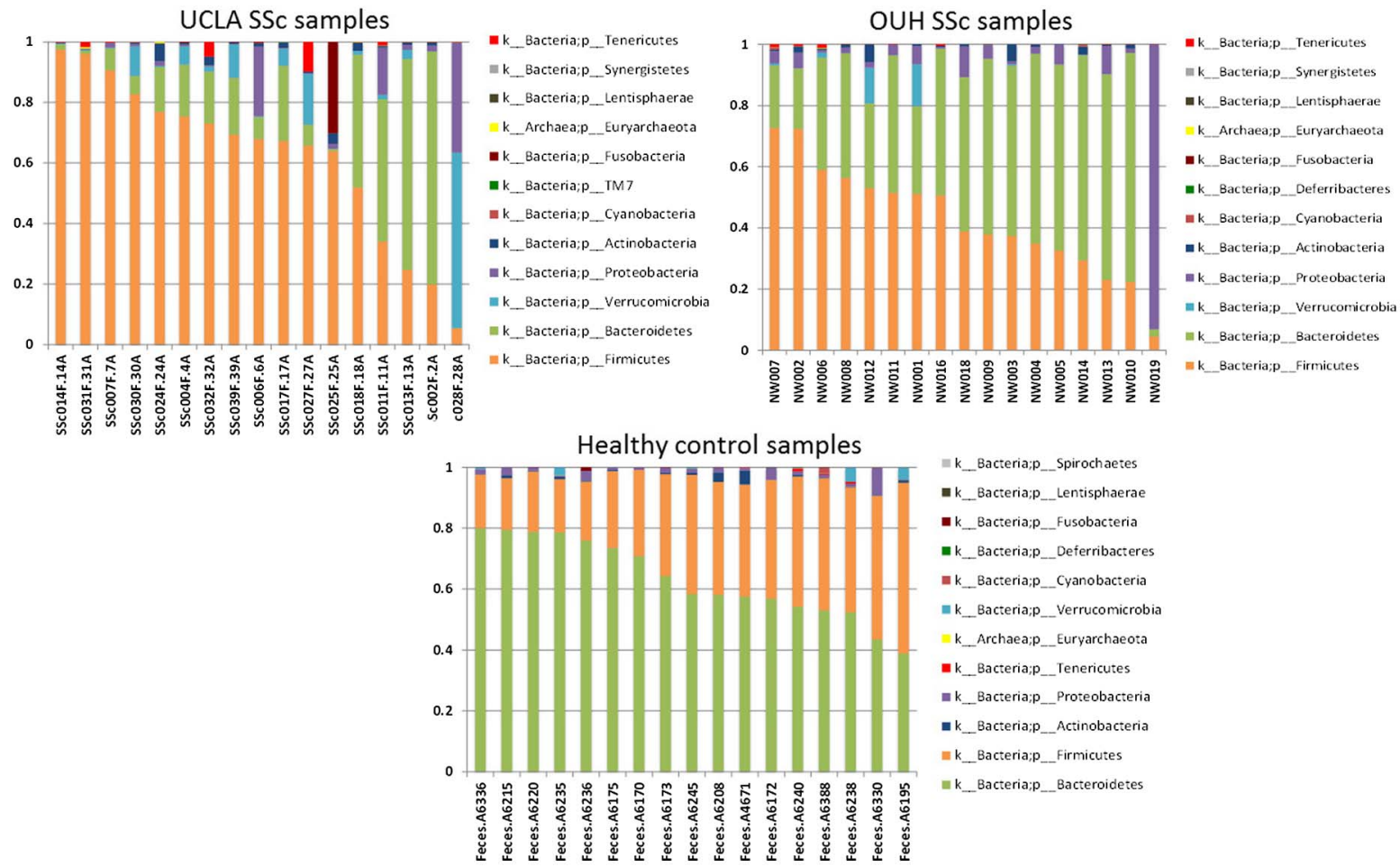

Figure 2 Microbial composition at the phylum level in UCLA-SSc samples (top left), OUH-SSc samples (top right) and healthy samples (bottom left). Legend provides colour coding specific to each phylum. OUH, Oslo University Hospital; SSc, systemic sclerosis; UCLA, University of California, Los Angeles.

more significant genus-level differences than the analysis comparing the OUH-SSc and healthy control cohorts.

Commensal genera such as Faecalibacterium (UCLA), Clostridium (OUH) and Bacteroides (UCLA, OUH) were depleted in patients with SSc; whereas, Fusobacterium (UCLA), Ruminococcus (UCLA) and the uncommon $\gamma$-Proteobacteria, Erwinia (UCLA), were enriched in patients with SSc, compared with health controls. Consistent with our prior study, ${ }^{10}$ Lactobacillus (considered to be a commensal genus ${ }^{17}$ ) was found in greater abundance in patients with SSc compared with controls in the UCLA and OUH cohorts (figures 3 and 4).

When comparing the UCLA-SSc and OUH-SSc cohorts, the LefSe analysis revealed that specific commensal genera, including Faecalibacterium and Bacteroides, were significantly more abundant in the OUH-SSc cohort patients compared with the UCLA-SSc cohort patients (fold change scores of 4.85 and 3.75, respectively).

\section{Specific microbial genera and species are associated with SSc-GIT symptoms}

Differential expression analysis for sequence count data multivariate analysis identified specific microbial genera associated with SSc-GIT symptom severity based on the scores for the total GIT 2.0 and individual domains (eg, constipation, diarrhoea or distension/bloating). For the total score and each domain, patients were dichotomised into low (none-to-mild) or high (moderate-to-severe) GIT symptom severity groups, and the fold change and q-values for organisms reaching significance were tabulated (see online supplementary table S1). The frequency of distribution of patients in each disease severity category were fairly well balanced ( $\mathrm{N}$ for low vs high groups): total score (18 vs 16); constipation (16 vs 18); distention/bloating ( 8 vs 26 ) and diarrhoea (18 vs 15 ).

In both SSc cohorts, Clostridium was more abundant in patients with low GIT symptom severity (for the total GIT score and the bloating/distension domain) compared with patients with high GIT symptom severity (figure 5 and see online supplementary table S1). Lactobacillus was more abundant in patients with none-to-mild constipation compared with patients with moderate-to-severe constipation. In contrast, Prevotella was more abundant in patients with moderate-to-severe GIT symptom severity (bloating/distention domain, diarrhoea domain) compared with patients with none-to-mild GIT symptom severity.

\section{DISCUSSION}

Consistent with our prior findings, ${ }^{10}$ the present study demonstrated that the SSc disease state is associated with alterations in the GIT microbial consortium. Using data from two independent SSc cohorts, we found that specific faecal microbial taxa were enriched or depleted among patients with SSc compared with healthy controls. We also demonstrated that specific taxa were associated with severity of GIT symptoms in both SSc cohorts.

In terms of phylum-level differences, the relative abundance of Bacteroidetes was significantly decreased in the 
Figure 3 Genus-level taxa associated with UCLA-SSc cohort patients versus healthy particiapnts. LefSe multivariate analysis was used to identify significant associations $(q<0.1)$, and LDA was used to calculate the effect size for these associations. Negative and positive effect sizes denote genera decreased (blue) or increased (red) in patients with SSc, respectively. All genera with an absolute LDA score $>2.5$ were included in this figure. LDA, linear discriminant analysis; LefSe, linear discriminant analysis effect size; SSc, systemic sclerosis; UCLA, University of California, Los Angeles.
Figure 4 Genus-level taxa patients versus healthy participants. LefSe multivariate analysis was used to identify significant associations $(q<0.1)$, and LDA was used to calculate the effect size for these associations. Negative and positive effect sizes denote genera decreased (blue) or increased (red) in patients with SSc, respectively. All genera with an absolute LDA score $>2.5$ were included in this figure. LDA, linear discriminant analysis; LefSe, linear discriminant analysis effect size; OUH, Oslo University Hospital; SSc, systemic sclerosis. associated with OUH-SSc cohort

p__Bacteroidetes;g__Bacteroides

p_Firmicutes;g_Faecalibacterium -

p__Bacteroidetes;g__Parabacteroides

p__Firmicutes;g__Lachnospira-

p__Proteobacteria;g_Sutterella-

p__Deferribacteres;g__Mucispirillum -

p__Bacteroidetes;g__Alistipes -

p__Proteobacteria;g__Campylobacter-

p__Firmicutes;g__Anaerobacillus -

p__Firmicutes;g__Megasphaera-

p__Proteobacteria;g__Herminiimonas-

p__Firmicutes;g_Vagococcus -

p__Firmicutes;g__Parvimonas -

p__Proteobacteria;g__Gluconacetobacter-

p__Firmicutes;g_Anaerococcus -

$\mathrm{p} \_$_Actinobacteria;g__Collinsella-

p__Proteobacteria;g__Morganella

p_Firmicutes;g__Bulleidia-

p__Proteobacteria;g_Acinetobacter-

p__Firmicutes;g__Pediococcus-

p_Firmicutes;g_Streptococcus -

p__Firmicutes;g__Dorea-

p__Proteobacteria;g_Erwinia-

p_Fusobacteria;g_Fusobacterium -

p__Firmicutes;g__Blautia-

p_Firmicutes;g__Ruminococcus -

p__Verrucomicrobia;g_Akkermansia-
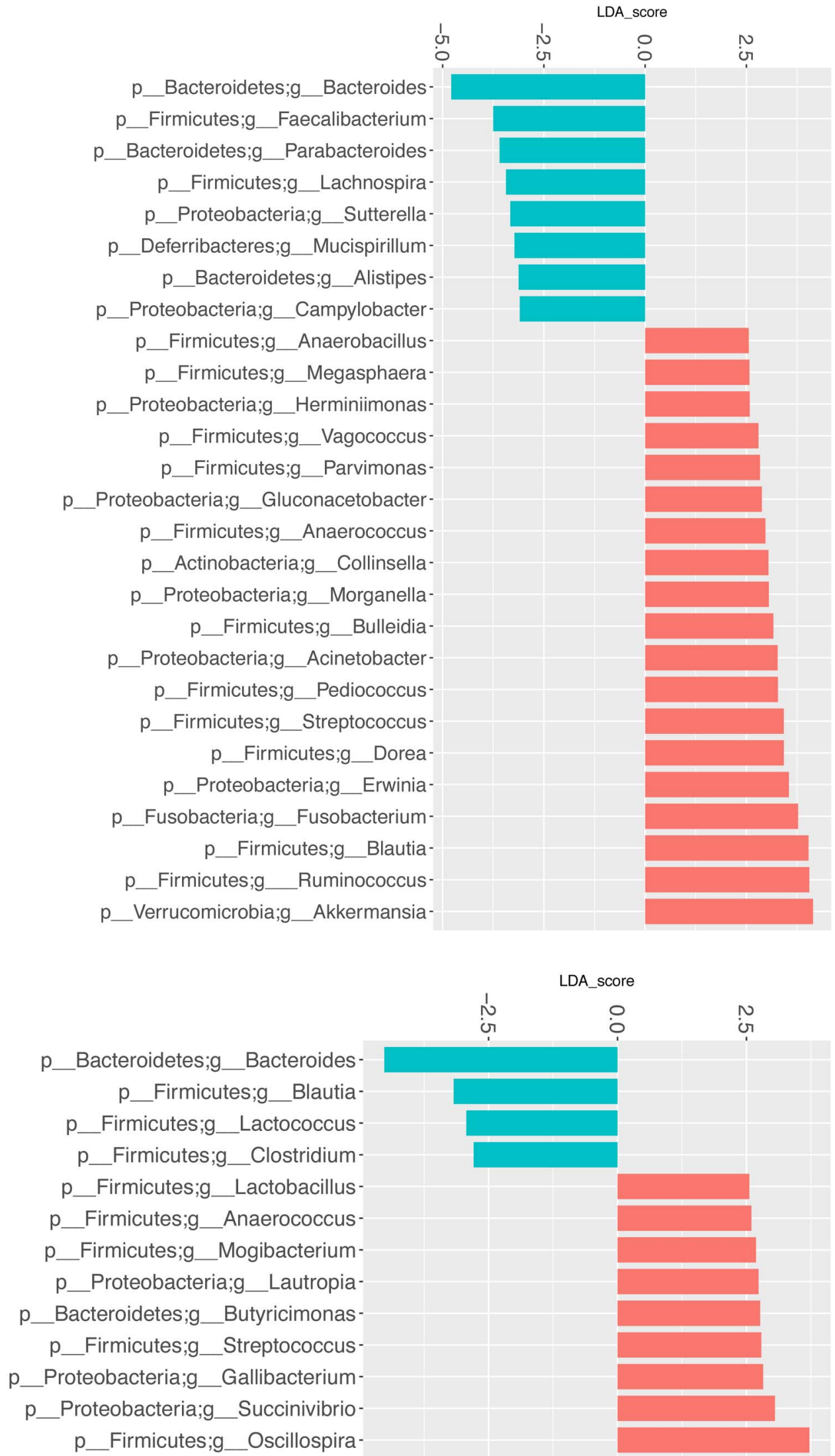

.

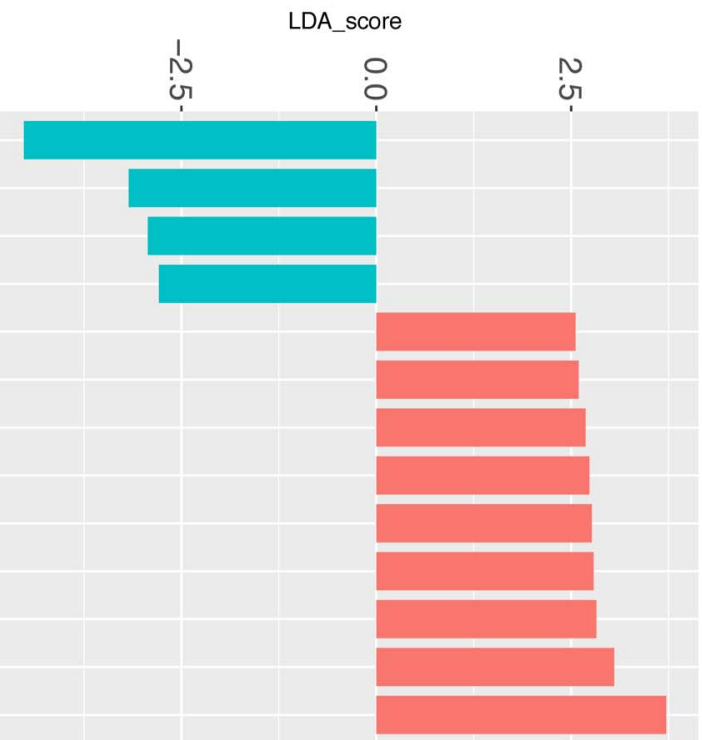

UCLA and OUH-SSc cohorts compared with the healthy control cohort. This finding is of interest in light of the recent evidence suggesting that the ratio of Firmicutes to Bacteroidetes may have an important effect on human health. ${ }^{18} 19$ Patients with SSc in the UCLA cohort had the lowest proportion of Bacteroidetes (21.3\%), followed by patients with SSc in the OUH cohort $(45.0 \%)$ and healthy controls $(63.2 \%)$. 


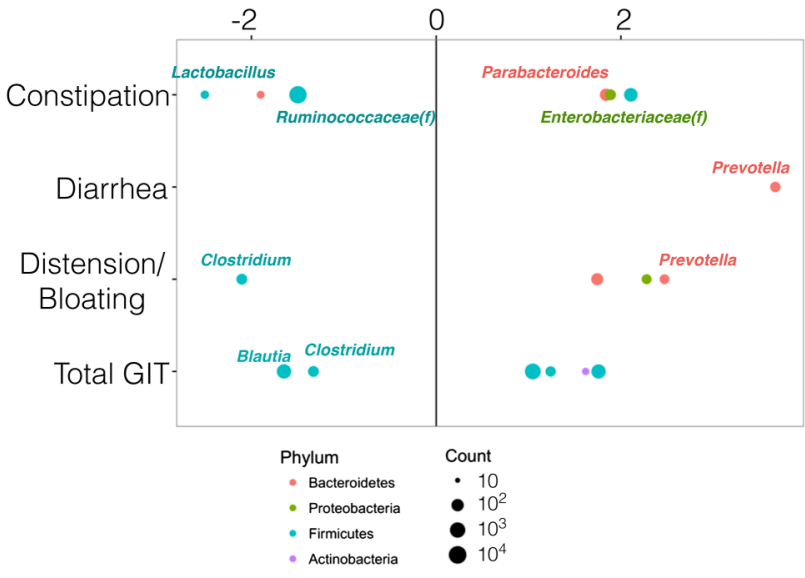

Figure 5 Bacterial taxa associated with GIT disease score and domains. Patients were dichotomised into low (none-to-mild) or high (moderate to severe) disease severity groups for the total GIT 2.0 score and its individual domains (constipation, diarrhoea or distension/bloating). DESeq2 multivariate analysis was used to identify microbial taxa significantly associated with low versus high groups (online supplementary table S1), and calculate the fold change between groups. Negative fold change scores (log2) denote organisms decreased in high disease severity groups; whereas, positive fold change scores denote organisms increased in high disease severity groups. Legend provides colour code of bacterial taxa at the phylum level; " $f$ " denotes family-level taxa. The size of the coloured dots represents the square root of the absolute mean counts of the OTUs at the genus level. DESeq2, differential expression analysis for sequence count data; GIT, gastrointestinal tract; OUT, operational taxonomic unit.

Consistent with the observed decrease in Bacteroidetes, lower level taxonomic analysis revealed that both $\mathrm{SSc}$ cohorts had significantly lower levels of the commensal genera Bacteroides, which is thought to protect the host from mucosal inflammation and against colonisation of pathogenic species. Low relative abundance of Bacteroides is associated with increased disease activity in other chronic inflammatory diseases, such as Crohn's disease (CD).$^{20}$ The fold change scores for the UCLA-SSc and OUH-SSc cohorts relative to controls was nearly 5 signifying a substantial shift in this genus in the SSc disease state.

Concordant with our prior study using colonic lavage specimens, ${ }^{10}$ we found that Faecalibacterium (UCLA) and Clostridium (OUH) were also depleted in SSc. Low abundance of these genera is associated with increased disease activity in IBD. ${ }^{21-23}$ As Clostridium species have been found to induce the expansion of regulatory $T$ cells, ${ }^{24}$ it is tempting to speculate that depletion of these taxa may reduce the number of regulatory $T$ cells, which may play a role in SSc pathogenesis. ${ }^{25}$ It is important to note, however, that species within this genus vary in terms of their metabolic properties. Future studies may explore the relationship between the abundance of specific species within the Clostridium genus and levels of circulating regulatory $\mathrm{T}$ cells.
In line with the potential immunoregulatory properties of Clostridium, we found that increased abundance of this genus was associated with decreased GIT symptoms (for the total GIT symptom severity score and the bloating/distension domain score). Our prior study also reported a link between Clostridium and GIT symptoms. ${ }^{10}$ If future studies demonstrate that Clostridium attenuates local or systemic inflammation in SSc (through effects on $\mathrm{T}$ regulatory cells), therapy aimed at increasing the abundance or activity of species in this genus may improve SSc disease activity.

In contrast, Prevotella was more abundant in patients with more severe GIT symptoms (bloating/distention domain, diarrhoea domain scores). This genus appears to have effects on intestinal $\mathrm{T}$ helper 17 cells, ${ }^{26}$ and it has been found to be increased in faecal samples from patients with $\mathrm{CD},{ }^{27}{ }^{28}$ and new-onset rheumatoid arthritis. $^{29}$

Also consistent with our prior study, the abundance of the pathobiont genera, Fusobacterium, and uncommon $\gamma$-Proteobacteria (eg, Erwinia) were higher in the UCLA-SSc faecal samples compared with controls. Fusobacterium species are increased in patients with CD compared with healthy controls and have been mechanistically proven to enhance proinflammatory responses. ${ }^{30} 31$

We are intrigued by the increase of Lactobacillus in the UCLA-SSc and OUH-SSc cohorts compared with healthy controls. This finding has been observed now in three different SSc cohorts (UCLA (colonic lavage specimens, faecal specimens), OUH (faecal specimens), Swedish cohort (faecal specimens)). ${ }^{10} 32$ We do not attribute this expansion to probiotic use. First, due to the exceptionally larger scale of the intestinal microbiome, ingestion of lactobacillus-bearing probiotics does not measurably alter faecal microbial composition. ${ }^{33}$ Second, very few patients in the present cohorts were taking probiotics on a regular basis, and none were taking probiotics within 3 weeks of the stool collection. Deemed a beneficial commensal genus by several host inflammatory and physiological end points in animal models, its abundance is typically reduced in chronic inflammatory human diseases.. ${ }^{34}$ We further note that members of this genus and their products alter the broader intestinal microbial ecosystem gene expression and function, which may be an intermediary of their action on systemic host phenotypes. ${ }^{33} 35$ Since many patients with SSc consume commercial probiotic supplements that commonly include Lactobacillus species, the utility of Lactobacillus as a biomarker or potential intervention in patients deficient for this genus warrants further study.

The overall extent of dysbiosis appeared greatest in the patients with SSc from UCLA compared with patients with SSc from the OUH cohort. Possible explanations for this disparity include different SSc phenotypes between the cohorts. For instance, more patients with SSc in the UCLA cohort had ILD than in the OUH cohort. Accumulating evidence suggests that GIT microbiota plays a central role in regulating immune 
responses in pulmonary disease, such as asthma. ${ }^{36}$ It is possible that patients with SSc-ILD have distinct microbiota features compared with patients with SSc without ILD. Indeed, a recent analysis by Andréaasen et $a l^{2}$ found that among patients with SSc from a single-centre cohort, the extent of GIT dysbiosis was more severe in patients with ILD compared with patients without ILD. Furthermore, although there was a similar ratio of patients with diffuse versus limited cutaneous disease in each SSc cohort, there may have been subtle differences in the peak severity of cutaneous sclerosis between the two cohorts.

In addition to SSc disease phenotype, genetic variation may account for some of the observed differences in severity of dysbiosis between the two SSc cohorts. The majority of the OUH-SSc cohort was Caucasian with a genetic Norwegian background; whereas the UCLA cohort was more ethnically diverse. A study for ulcerative colitis reported that ethnicity stratifies a small proportion $(7.5 \%)$ of observed variation in $\beta$ diversity. ${ }^{37}$ Dietary variation may also contribute to the differences observed between the two SSc cohorts.

In contrast with a prior research, ${ }^{32}{ }^{38}$ faecal calprotectin concentrations were not elevated in the majority of patients with SSc (from the UCLA cohort), although different kits/protocols may have been used in prior studies. While accumulating evidence suggests that faecal calprotectin is seen in inflammatory intestinal diseases (eg, IBD, colorectal cancer), future studies are needed to determine whether this protein is a marker of disease activity or the extent of dysbiosis in SSc.

The findings of the present study should be considered within the context of certain limitations. First, the present study is cross-sectional. It is unclear whether the relationships observed between specific genera and GIT symptoms are causational and/or persist with time. Second, the sample size for both SSc cohorts is small. Despite the small sample size, we observed several significant associations consistent with the associations reported in our prior study, ${ }^{10}$ suggesting that the present findings are unlikely to be due to chance alone. Third, the same healthy control cohort was used for the UCLA and OUH-SSc cohorts. It may have been more ideal to use a Norwegian healthy control cohort for the OUH-SSc analyses to control for genetic and dietary variation.

In addition, it may be prudent to include a measure of GIT transit in future SSc-GIT microbiome studies. This study did not assess GIT transit as there is no valid measure of GIT transit in SSc; however, diarrhoea and constipation are intrinsically related to GIT transit. Future studies of this nature may consider using potential surrogate measures of GIT transit, such as wireless capsule endoscopy. Finally, although it is a challenge to quantify dietary patterns due to patient recall bias, future studies could also consider the impact of diet on microbial composition in SSc. Accordingly, a distinct study design will be required to assess the differential microbiota associated with disease state or phenotypic progression.
The present study also has important strengths. By studying two independent SSc cohorts, we have minimised the risk of type 1 error that often ensues in discovery cohort analyses of this nature. In addition, by performing all of the sequencing analyses simultaneously, we have eliminated the possibility of a batch effect. We also took substantial caution to ensure that all patients withheld medications, such as antibiotics and probiotics, at least 3 weeks prior to the stool collection, by verifying medication lists three times in the month preceding the collection.

To conclude, this study identified bacterial genera associated with SSc using sophisticated sequencing analyses of faecal specimens. Many of the genus-level gains and losses appreciated in the faecal specimens examined in the present study were also observed in our prior study using colonic-mucosal specimens from the same patients with SSc (but different control patients). ${ }^{10}$ Using two independent SSc cohorts, this study also uncovered relationships between specific genera and severity of SSc-GIT symptoms, which merit further investigation. While larger studies are needed to validate and expand on these findings, the present study is the first to characterise the lower GIT microbiota in two geographically and ethnically distinct SSc cohorts.

\section{Author affiliations}

${ }^{1}$ Department of Medicine, University of California, Los Angeles, David Geffen School of Medicine, Los Angeles, California, USA

${ }^{2}$ Department of Rheumatology, Oslo University Hospital, Oslo, Norway

${ }^{3}$ Department of Pathology and Laboratory Medicine, University of California, Los Angeles, David Geffen School of Medicine, Los Angeles, California, USA ${ }^{4}$ Division of Surgery, Inflammatory Diseases and Transplantation, Norwegian PSC Research Center, Oslo University Hospital Rikshospitalet, Oslo, Norway ${ }^{5}$ Division of Surgery, Inflammatory Diseases and Transplantation, Research Institute of Internal Medicine, Oslo University Hospital Rikshospitalet, Oslo, Norway

${ }^{6}$ Faculty of Medicine, Institute of Clinical Medicine, University of Oslo, Oslo, Norway

Acknowledgements The authors thank the patient volunteers for their participation in this study.

Contributors ERV provided substantial contributions to study conception and design; collection of data; analysis and interpretation of data; drafting the article; approval of the final manuscript. ERV is responsible for the overall content as a guarantor. A-MH-V provided substantial contributions to study conception and design; collection of the data; revising the manuscript critically for important intellectual content; approval of the final manuscript. A-MH-V is responsible for the overall content as a guarantor. Y-LC provided substantial contributions to study conception and design; analysis and interpretation of the data; revising the manuscript critically for important intellectual content; approval of the final manuscript. JPJ, KT, EAM and PJC provided substantial contributions to study conception and design; revising the manuscript critically for important intellectual content; approval of the final manuscript. JRH, MK and ØMi provided substantial contributions to the analysis and interpretation of data; revising the manuscript critically for important intellectual content; approval of the final manuscript. VL provided substantial contributions to the analysis and interpretation of data; analysing the biospecimens; revising the manuscript critically for important intellectual content; approval of the final manuscript. LC, JL and ØMo provided substantial contributions to the analysis and interpretation of data; revising the manuscript critically for important intellectual content; approval of the final manuscript. JB provided substantial contributions to study conception 
and design, the analysis and interpretation of data; revising the manuscript critically for important intellectual content; approval of the final manuscript.

Funding This work was supported by grants from CURE: Digestive Disease Research Center supported by NIH grant P30DK41301-Pilot and Feasibility (ERV); NIH P0-1 DK46763 (JB) and UL1TR001881 (JB); The Unger-Vetlesen Foundation (A-MH-V); The Norwegian Women's Public Health Association (A-MH-V); Iris Cantor-UCLA Women's Health Center Executive Advisory Board (KT); NCATS UCLA CTSI UL1TR000124 (KT); NIH P50 DK064539 (EAM); Health Authority of South-Eastern Norway 2016067 (MK); and Norwegian Research Council 240787/F20 (JRH).

Disclaimer The views expressed in the submitted article are the authors' own and not an official position of the institution or funder.

Competing interests None declared.

Ethics approval UCLA Institutional Review Board (IRB) and the Regional Committee of Health and Medical Research Ethics in Norway.

Provenance and peer review Not commissioned; externally peer reviewed.

Data sharing statement No additional data are available.

Open Access This is an Open Access article distributed in accordance with the Creative Commons Attribution Non Commercial (CC BY-NC 4.0) license, which permits others to distribute, remix, adapt, build upon this work noncommercially, and license their derivative works on different terms, provided the original work is properly cited and the use is non-commercial. See: http:// creativecommons.org/licenses/by-nc/4.0/

\section{REFERENCES}

1. Lock G, Holstege A, Lang B, et al. Gastrointestinal manifestations of progressive systemic sclerosis. Am J Gastroenterol 1997;92:763-71.

2. Sallam H, McNearney TA, Chen JD. Systematic review: pathophysiology and management of gastrointestinal dysmotility in systemic sclerosis (scleroderma). Aliment Pharmacol Ther 2006:23:691-712.

3. Marie I, Ducrotté P, Denis $\mathrm{P}$, et al. Small intestinal bacterial overgrowth in systemic sclerosis. Rheumatology 2009;48:1314-19.

4. Franck-Larsson K, Graf W, Rönnblom A. Lower gastrointestinal symptoms and quality of life in patients with systemic sclerosis: a population-based study. Eur J Gastroenterol Hepatol 2009:21:176-82.

5. Bodukam V, Hays RD, Maranian P, et al. Association of gastrointestinal involvement and depressive symptoms in patients with systemic sclerosis. Rheumatology (Oxford) 2011;50:330-4.

6. Frank DN, Amand ALS, Feldman RA, et al. Molecular-phylogenetic characterization of microbial community imbalances in human inflammatory bowel diseases. Proc Natl Acad Sci USA 2007:104:13780-5.

7. Fava $F$, Danese S. Intestinal microbiota in inflammatory bowel disease: friend of foe? World J Gastroenterol 2011;17:557-66.

8. Presley LL, Ye J, Li X et al. Host-microbe relationships in inflammatory bowel disease detected by bacterial and metaproteomic analysis of the mucosal-luminal interface. Inflamm Bowel Dis 2012;18:409-17.

9. McHardy IH, Goudarzi M, Tong M, et al. Integrative analysis of the microbiome and metabolome of the human intestinal mucosal surface reveals exquisite inter-relationships. Microbiome 2013;1:17.

10. Volkmann ER, Chang YL, Barroso N, et al. Association of systemic sclerosis with a unique colonic microbial consortium. Microbiome 2016:68:1483-92.

11. van den Hoogen F, Khanna D, Fransen J, et al. 2013 classification criteria for systemic sclerosis: an American College of Rheumatology/European League against Rheumatism collaborative initiative. Arthritis Rheum 2013;65:2737-47.

12. Tsuda A, Suda W, Morita $\mathrm{H}$, et al. Influence of proton-pump inhibitors on the luminal microbiota in the gastrointestinal tract. Clin Trans/ Gastroenterol 2015;6:e89.

13. Tong M, Jacobs JP, McHardy IH, et al. Sampling of intestinal microbiota and targeted amplification of bacterial 16S rRNA genes for microbial ecologic analysis. Curr Protoc Immunol 2014;107:7.41.1-11.

14. Khanna D, Hays RD, Maranian $P$, et al. Reliability and validity of the University of California, Los Angeles scleroderma clinical trial consortium gastrointestinal tract instrument. Arthritis Care Res 2009;61:1257-63

15. Benjamini $\mathrm{Y}$, Hochberg $\mathrm{Y}$. Controlling the false discovery rate: a practical and powerful approach to multiple testing. J R Stat Soc Series B 1995;57:289-300

16. Goh NS, Desai SR, Veeraraghavan S, et al. Interstitial lung disease in systemic sclerosis: a simple staging system. Am J Respir Crit Care Med 2008;177:1248-54.

17. Kostic AD, Xavier RJ, Gevers D. The microbiome in inflammatory bowel disease: current status and the future ahead. Gastroenterology 2014;146:1489-99.

18. Ley RE, Turnbaugh PJ, Klein S, et al. Microbial ecology: human gut microbes associated with obesity. Nature 2006;444:1022-3.

19. Mariat D, Firmesse O, Levenez F, et al. The Firmicutes/ Bacteroidetes ratio of the human microbiota changes with age. BMC Microbiol 2009;9:123.

20. Schaffler $\mathrm{H}$, Herlemann DP, Alberts $\mathrm{C}$, et al. Mucosa-attached bacterial community in Crohn's disease coheres with the clinical disease activity index. Environ Microbiol Rep 2016. doi: 10.1111/ 1758-2229.12411 [Epub: 26 May 2016].

21. Sokol H, Pigneur B, Watterlot L, et al. Faecalibacterium prausnitzii is an anti-inflammatory commensal bacterium identified by gut microbiota analysis of Crohn disease patients. Proc Natl Acad Sci 2008;105:16731-6.

22. Varela E, Manichanh $\mathrm{C}$, Gallart M, et al. Colonisation by Faecalibacterium prausnitzii and maintenance of clinical remission in patients with ulcerative colitis. Aliment Pharmacol Ther 2013;38:151-61.

23. Prosberg M, Bendtsen F, Vind I, et al. The association between the gut microbiota and the inflammatory bowel disease activity: a systematic review and meta-analysis. Scand J Gastroenterol 2016;51:1407-15.

24. Atarashi K, Tanoue $\mathrm{T}$, Oshima $\mathrm{K}$, et al. Treg induction by a rationally selected mixture of Clostridia strains from the human microbiota. Nature 2013;500:232-6.

25. Slobodin G, Rimar D. Regulatory T cells in systemic sclerosis: a comprehensive review. Clin Rev Allergy Immunol 2017;52:194-201.

26. Maeda $\mathrm{Y}$, Kurakawa $\mathrm{T}$, Umemoto $\mathrm{E}$, et al. Dysbiosis contributes to arthritis development via activation of autoreactive T cells in the intestine. Arthritis Rheumatol 2016;68:2646-61.

27. Benjamin JL, Hedin CR, Koutsoumpas A, et al. Smokers with active Crohn's disease have a clinically relevant dysbiosis of the gastrointestinal microbiota. Inflamm Bowel Dis 2012;18:1092-100.

28. De Cruz P, Kang S, Wagner J, et al. Association between specific mucosa-associated microbiota in Crohn's disease at the time of resection and subsequent disease recurrrence: a pilot study. $J$ Gastroenterol Hepatol 2015;30:268-78.

29. Scher JU, Sczesnak A, Longman RS, et al. Expansion of intestinal Prevotella copri correlates with enhanced susceptibility to arthritis. Elife 2013;2:e01202.

30. Gevers D, Kugathasan S, Denson LA, et al. The treatment-naive microbiome in new-onset Crohn's disease. Cell Host Microbe 2014;15:382-92.

31. Strauss J, Kaplan GG, Beck PL, et al. Invasive potential of gut mucosa-derived Fusobacterium nucleatum positively correlates with IBD status of the host. Inflamm Bowel Dis 2011;17:1971-8.

32. Andréasson K, Alrawi Z, Persson A, et al. Intestinal dysbiosis is common in systemic sclerosis and associated with gastrointestinal and extraintestinal features of disease. Arthritis Res Ther 2016;18:278.

33. McNulty NP, Yatsunenko T, Hsiao A, et al. The impact of a consortium of fermented milk strains on the gut microbiome in gnotobiotic mice and monozygotic twins. Sci Transl Med 2011;3:106ra106

34. Walter J. Ecological role of lactobacilli in the gastrointestinal tract: implications for fundamental and biomedical research. Appl Environ Microbiol 2008;74:4985-96.

35. Tillisch K, Labus J, Kilpatrick L, et al. Consumption of fermented milk product with probiotic modulates brain activity. Gastroenterology 2013;144:1394-401.

36. Samuelson DR, Welsh DA, Shellito JE. Regulation of lung immunity and host defense by the intestinal microbiota. Front Microbio 2015;6:1085.

37. Mar JS, LaMere BJ, Lin DL, et al. Disease severity and immune activity relate to distinct interkingdom gut microbiome states in ethnically distinct ulcerative colitis patients. MBio 2016;7: e01072-16.

38. Marie I, Leori AM, Menard JF, et al. Fecal calprotectin in systemic sclerosis and review of the literature. Autoimmun Rev 2015;14:547-54. 